\title{
Distributed Photonic Instrumentation for Power System Protection and Control
}

\author{
Philip Orr, Grzegorz Fusiek, Paweł Niewczas, Campbell D. Booth, Adam Dyśko, Fumio Kawano, \\ Members IEEE, Tomonori Nishida and Phil Beaumont, Senior Member IEEE
}

\begin{abstract}
We report on recent developments in hybrid optical protection schemes that make use of passive fibre Bragg grating (FBG) based transducers for the distributed measurement of voltage and current. In addition to the details of the hybrid optical sensor technology, and its full integration with a commercial busbar protection relay, we report on the first-time laboratory demonstration of a centralized three-ended unit protection scheme featuring hybrid optical sensors, capable of passive and wide-area coverage. Furthermore, we discuss the impact of this technology on present and future protection applications that may be improved or enabled by the proposed scheme.
\end{abstract}

Index Terms-power system protection, optical fiber sensors, distributed protection, smart grids

\section{INTRODUCTION}

$\mathrm{C}$ OONVENTIONAL unit type protection schemes rely on the communication of measured currents and/or voltages between relays at different locations. In most cases this continuous communication of sampled values, phasors, or Boolean flags is transmitted digitally using optical fiber or other media [1]. In this configuration, the protection relays are always situated close to the measurement points, and additional relays are required for non-local measurements. Differential schemes based on this historical approach are therefore limited in flexibility and extensibility by the communication bandwidth, the requirement for local power at each measurement point, and the size, weight and insulation requirements of conventional instrument transformers. The latency of the communications link and the requirement to encode and decode the communicated data can also result in the communications system making a significant contribution to the operating time of the protection system.

Optical sensing is an established technology that provides solutions to a number of measurement problems posed by adverse environmental conditions, such as those commonly encountered in the oil and gas sector, nuclear industry and electrical power generation and distribution [2-4]. In many

Manuscript received $20^{\text {th }}$ October, 2012. This work was supported in part by the Engineering and Physical Sciences Research Council and in part by Toshiba Corporation.

P. Orr, G. Fusiek, P. Niewczas, C.D. Booth and A. Dyśko are with the Institute for Energy and Environment, University of Strathclyde, Glasgow, G1 1XW, UK (e-mail: philip.orr@strath.ac.uk).

F. Kawano and T. Nishida are with Toshiba Corporation, Tokyo, 1058001, Japan (e-mail: fumio.kawano@toshiba.co.jp).

P. Beaumont is with Toshiba International (Europe), London, UK. cases, conditions in these environments are such that conventional electronic sensors cannot operate to the required standards. Fiber-optic sensing is a high-performance measurement technology incorporating transducers and sensor networks that are passive, compact, chemically inert, immune to electromagnetic interference, and able to be interrogated over long distances [5].

Optical current transducers (OCTs) are now relatively well established and are developed by a range of manufacturers for their passive and highly accurate measurement of electrical current [6,7]. Presently, the most successful design is based on either spun or annealed optical fiber (to reduce shape-induced linear birefringence) that is coiled around the conductor or current path. The contour integral of the circulating magnetic field then yields a polarimetric or interferometric measurement of enclosed current based on the Faraday effect [8] that is immune to the influence of external or stray fields. Optical voltage transducers (OVTs) have also been developed, primarily based upon either the electro-optic (Pockels) effect or the piezoelectric effect $[7,9]$. Both established OCTs and OVTs rely on interferometric or polarimetric measurement techniques, and thus the distance from interrogator to measurement point cannot be greater than around 100 metres in most cases. Additionally, as with conventional electrical transducers, it is not possible to discriminate between superimposed sensor responses, and thus serial multiplexing is not possible. For these reasons, wide-area coverage, measurement over long distances, and high numbers of sensors are not presently achieved by optical current or voltage measurement schemes. Therefore, present commercial optical transducers for the power industry offer gains in measurement quality (accuracy, rate, and immunity to interference), but cannot contribute to the development of novel protection architectures or applications.

In this paper, we demonstrate a scheme for optical measurement of voltage and current that is based on in-fiber point sensors. The proposed sensors are physically small and are able to be multiplexed, thus overcoming limitations in existing optical schemes and allowing the implementation of novel protection architectures that are not achievable either with present electrical or optical measurement techniques in the power industry. Such an approach has the potential to address the limitations of standard protection architectures by presenting, at a single location, data relating to voltage and/or current from multiple widespread locations on a network.

We first introduce in Section II the optical measurement technique and sensor network topology. Section III reports on collaborative work with Toshiba Corporation on the 
integration of this measurement technique with existing busbar protection relay hardware. The first full laboratory demonstration of the hybrid system is reported in Section IV, where the scheme is successfully configured to provide threeended unit protection over a wide area. Finally, in Section V we discuss the excellent potential of the approach to address a variety of present and future challenges in power system protection.

\section{FIBER-OPtIC SENSING SCHEME}

Current transducers may be classified [10] as: (i) conventional, i.e. utilizing resistive shunts or iron core or Rogowski type transformers; (ii) pure optical based on the Faraday effect as described in Section I; or (iii) extrinsic optical. It is sensors of the latter category that are proposed in this article, where an intermediate transducer is used to convert current into a secondary quantity - often strain - that is measurable by the all-optical transducer.

\section{A. Fiber Bragg gratings (FBGs)}

Fiber Bragg gratings (FBGs) are periodic perturbations of the refractive index along a fiber core, having peak optical reflection at a specific wavelength, known as the Bragg wavelength [11], and a typical physical length of 5-10 $\mathrm{mm}$. In sensor applications, their wavelength-encoding nature, coupled with their simple reflected spectra, means that FBGs are relatively easy to interrogate and multiplex, and are effectively immune to the problems of intensity fluctuations and attenuation [3]. For these reasons the FBG is now ubiquitous in the field of optical instrumentation $[4,12]$.

Peak optical reflection from FBGs occurs at a wavelength $\lambda$ equal to twice the grating period, i.e. at $\lambda / n=2 \Lambda$ where $n$ is the fiber refractive index and $\Lambda$ is the pitch of the grating. Thus, straining or compressing the fiber longitudinally at the location of the grating shifts up or down, respectively, the peak reflected wavelength. Illumination of the FBG by broadband light, and some form of peak wavelength detection and tracking, may therefore be employed to utilize the FBG as a strain sensor.

\section{B. FBG-based voltage and current sensors}

The authors have previously developed fiber-optic voltage and current point sensors, based on FBG technology, that have been applied successfully to power system plant diagnostics $[13,14]$. The complete optical sensor system has been shown to be capable of measuring dynamically changing signals and has been successfully used for detecting higher order voltage and current harmonics [15].

The hybrid voltage sensor employed in this work utilizes an FBG bonded to a multilayer piezoelectric stack, while the current sensor uses a small, high-bandwidth current transformer monitored by a dedicated voltage sensor as shown in Fig. 1. In both cases an FBG peak wavelength shift can be calibrated in terms of voltage or current, while for voltages or currents, a temperature measurement can also be performed simultaneously using the same sensors.

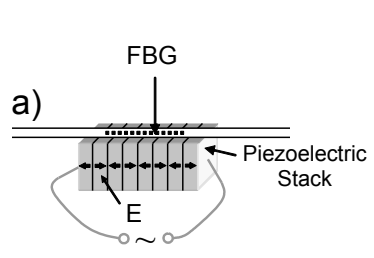

b)

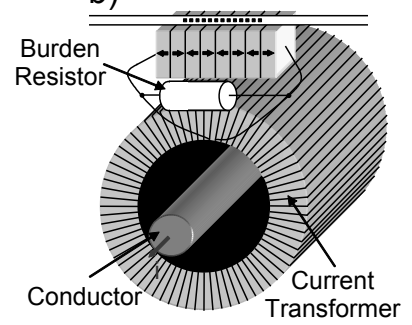

Fig. 1. (a) Hybrid piezoelectric voltage sensor. (b) Hybrid current sensor employing a voltage sensor and a current transformer.

It was demonstrated previously that these fiber-optic voltage and current sensors can be used for measuring variable frequency voltage and current waveforms for use in future aero-electric power systems [16]. Their use in simplified differential and distance protection schemes (implemented within the sensor interrogation unit) has also been reported in a preliminary form $[17,18]$. Most recently, the authors presented initial results showing retrofitting capabilities [19], and in this paper a comprehensive disclosure of this approach is provided.

\section{Optical sensor network topology and interrogation}

The generic architecture of an FBG sensor scheme is illustrated in Fig. 2. Light from an optical source is guided by fiber to an array of serially-multiplexed FBGs. Reflections from all FBGs are returned via a coupler to the interrogating device, at which the peak reflected wavelength from each sensor is extracted.

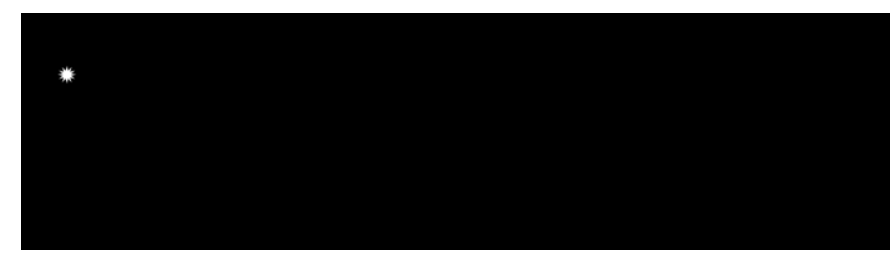

Fig. 2. A generic FBG measurement scheme illustrating the multiplexed and reflection-mode topology. $\lambda_{1}$ and $\lambda_{2}$ are peak reflected wavelengths.

In this work, we utilize a long-distance multiplexing FBG interrogator developed at the University of Strathclyde for the monitoring of subsea electrical submersible pumps (Fig. 3) [5].

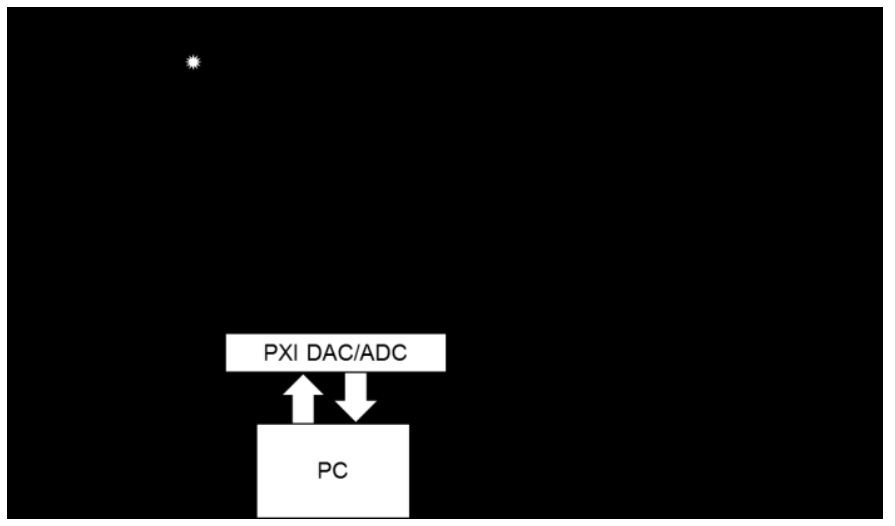

Fig. 3. Scanning filter based FBG interrogator utilized in this work. 
The general architecture of this scheme is commonly utilized in optical fiber sensor systems: a tunable filter is scanned over the bandwidth of the source and the photodetector output is sampled continuously. In this particular embodiment, extraction and tracking of FBG peaks is performed in software based on the recorded spectra. It should be noted that alternative interrogation schemes may be used. In general, present interrogation schemes possess a trade-off between the extensibility (number of sensors) and the performance (rate, accuracy, resolution). However, recently developed schemes are beginning to address this [20].

\section{INTEGRATION WITH PROTECTION RELAY}

We reported previously on the development of a platform for real-time communication of measured values between an optical sensor interrogator and a microprocessor based protection relay device [21]. Here, the method and verification of this integration is summarized briefly for completeness.

\section{A. Implementation of communications protocol}

In order to demonstrate the integration of multiplexed optical current measurement with protection hardware, the optical measurement scheme described above was configured to emulate the output of a Toshiba GRB100 bay unit [22]. In order to facilitate communication between the optical measurement scheme and the GRB100, Toshiba's bespoke communication protocol for interfacing between the relay central unit (CU) and associated bay units (BUs) was implemented within the optical measurement scheme. This allowed for direct communication with the GRB100 central unit by emulation of the BU output format. The optical scheme thus behaves as a BU by delivering sample-by-sample data relating to measured currents in the expected format. It should be noted that in future iterations of this technology, any output format may be implemented, including direct output of sampled values from numerous voltage and current sensors on to an IEC61850 process bus [23].

In the present embodiment, two multifunction National Instruments PXI cards are used, permitting acquisition of signals from optical current sensors and data communication between the relay and the sensor interrogation unit. The card sampling rate was adjusted such that 48 samples per period of the current waveform were acquired - in accordance with the GRB100 protocols. After encoding, the data for all measurements are transmitted via optical transceivers in response to synchronization signals generated by the GRB100 CU.

\section{B. Verification of real-time communication}

In order to verify the correct functioning of the communication interface, $\mathrm{AC}$ currents were applied to three optical current sensors simultaneously. The waveforms, as recorded by the optical scheme and on the GRB100 disturbance records, were then compared directly.

Fig. 4 illustrates the test configuration adopted. The scheme incorporates the interrogation scheme of Fig. 3, with the addition of three fiber-optic current sensors driven by an APTS3 current injection unit [24]. Following simultaneous optical interrogation of the three single-phase currents, the data is packaged appropriately for quasi-synchronous transmission that emulates three separate bay units.

It is clear from Figs. 5 and 6 that there is excellent agreement between waveforms either side of the communication interface. Small "smoothing" differences can be seen due to analog and digital filtering implemented within the relay.

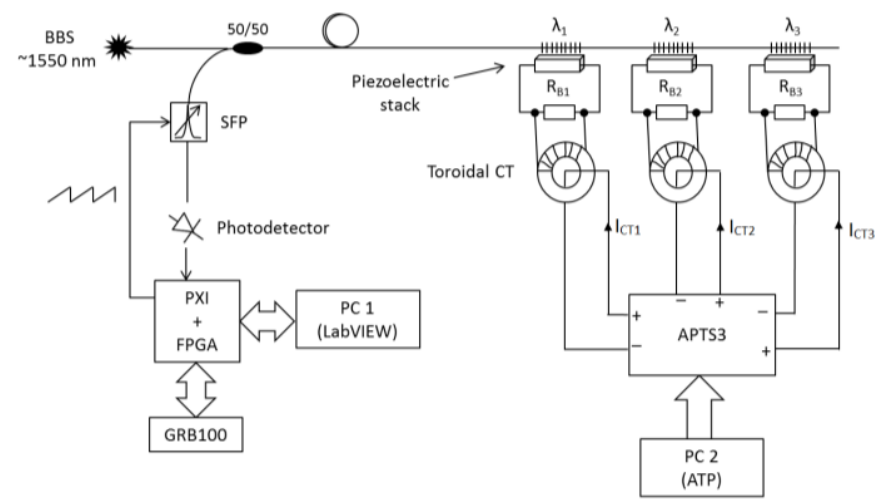

Fig. 4. Single-phase single-fiber laboratory test scheme using APTS3 current injection unit. $\lambda_{1-3}$ are sensor Bragg wavelengths, $\mathrm{R}_{\mathrm{B} 1-3}$ are burden resistors, BBS is broadband optical source, SFP is scanning optical filter.
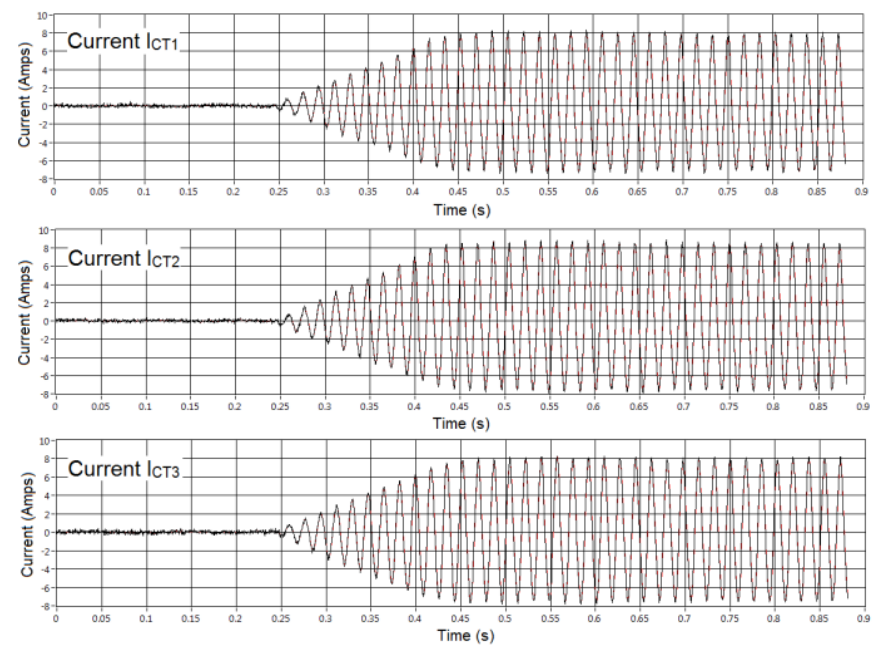

Fig. 5. Simultaneous measurement of three currents (generated in phase) according to the optical sensor raw data (black) and relay disturbance records (red).

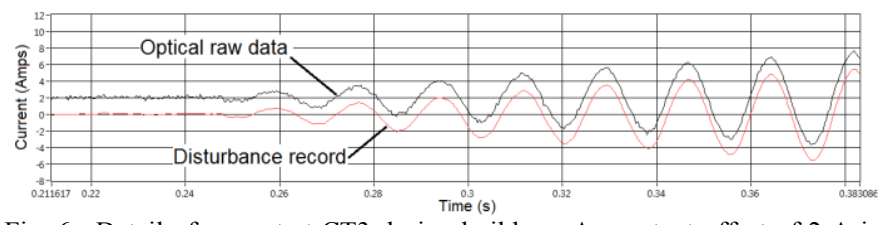

Fig. 6. Detail of current at CT3 during build up. A constant offset of $2 \mathrm{~A}$ is applied to the optical waveform (black) for visual clarity.

\section{LABORATORY DEMONSTRATION OF THREE-TERMINAL UNIT PROTECTION}

In order to demonstrate operation of the technique under realistic conditions, the integrated platform comprising a GRB100 relay and sensor interrogator (described in Section III) was applied to the protection of an operational section of Strathclyde's microgrid laboratory. The protection of a threeended circuit using a trio of optical sensors multiplexed on a 


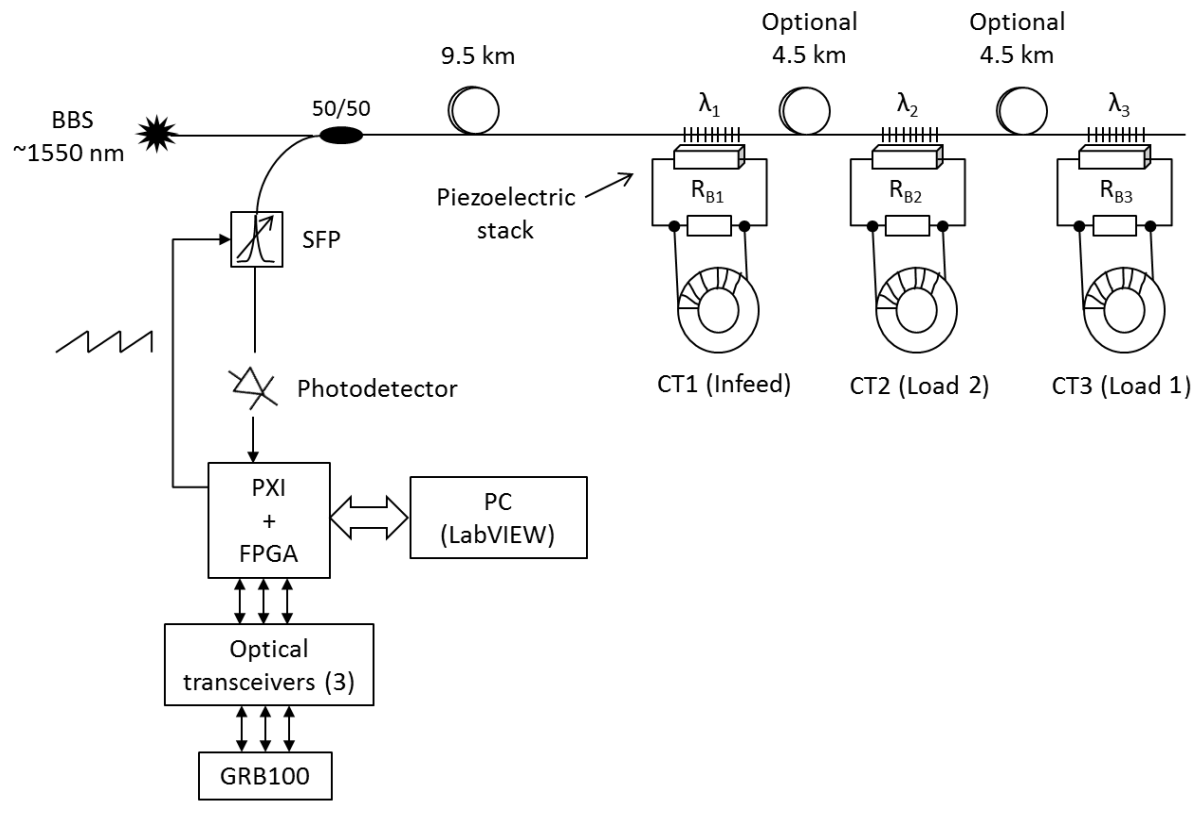

Fig. 8. Configuration of hybrid optical interrogator and relay comprising a GRB100 busbar protection relay central unit (Toshiba) and a scanning filterbased optical sensor interrogation scheme. Fiber reels are installed between sensor locations in order to demonstrate wide-area passive coverage of the protected zone. BBS is broadband source, SFP is scanning Fabry-Pèrot filter.

single fiber provides a full demonstration of the hybrid relay/interrogator in a laboratory environment, and a clear illustration of the potential for this scheme to implement widearea protection applications. In this section, the configurations of the protected electrical network, hybrid optical/GRB100 protection system, and fault-throwing mechanism are described, and thereafter the application of a range of internal and external fault scenarios is reported.

\section{A. Configuration of protected electrical network}

A microgrid laboratory was used to construct a singlephase, three-ended circuit comprising an infeed and two resistive loads. With reference to Fig. 7, the transducers were located after the current-limiting impedance (inductance) and directly before each load. In these experiments, purely resistive loads were used, each drawing approximately $10 \mathrm{~A}$ rms (at $230 \mathrm{~V}$ rms single phase).

In these tests, phase-earth faults are applied either within the protected zone (immediately prior to the splitting of the conductor path) or externally, immediately prior to the second load as indicated in Fig. 7. The fault-limiting inductance is manually configurable; by altering the series reactance of the infeed, internal fault levels of 30 and $70 \mathrm{~A}$ rms were configured. Both fault levels are utilized in the internal scenarios, whereas in order to protect certain components the external fault level was limited to $30 \mathrm{~A}$.

\section{B. Configuration of optical hybrid interrogator/relay}

Fig. 8 illustrates the architecture of the optical interrogation system and GRB100 protection relay to be applied to the electrical network. As detailed, the optical interrogation system is configured to continuously communicate measured current values to the GRB100 central unit on a sample by sample basis asynchronously as triggered by the GRB100 output. An FPGA system is configured as the intermediate buffer and transceiver between both schemes. Data is delivered to the central unit in the format expected from three bay units.

For this laboratory demonstration, data were transmitted to the relay on three separate optical channels (one measurement location per channel) in order to emulate a three-ended single phase busbar protection scheme that would conventionally require three separate GRB100 bay units (with associated local power sources and dedicated communication channels).

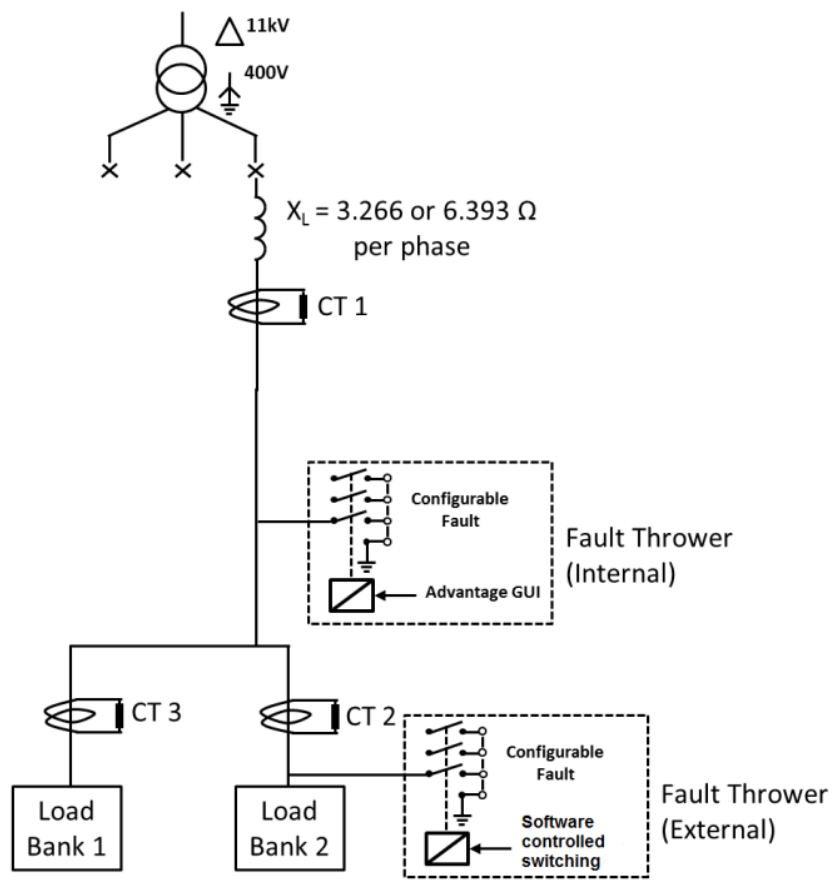

Fig. 7. Electrical configuration showing transducer CTs located after infeed inductance and prior to both resistive loads. 
The sensor wavelengths $\lambda_{1}, \lambda_{2}$ and $\lambda_{3}$ were situated at 1535 $\mathrm{nm}, 1545 \mathrm{~nm}$, and $1560 \mathrm{~nm}$ respectively, which is within the telecommunications $\mathrm{C}$-band to facilitate compatibility with standard telecommunication components. These sensor wavelengths are configured to correspond to CTs 1, 2 and 3 respectively in Fig. 7. Table 1 summarizes the position and wavelength of each transducer.

With reference to Table 2, during all tests, $9.5 \mathrm{~km}$ of singlemode fiber was installed between the interrogation system and CT1. Removable optical fiber lengths of $4.5 \mathrm{~km}$ are installed between CTs 1 and 2, and between CTs 2 and 3. These reels were installed for one batch of internal fault tests, and removed for another in order to demonstrate the increase in noise due to attenuation of optical power. During external faults, the $4.5 \mathrm{~km}$ sections were installed between sensors.

TABLE 1

DETAILS OF OPTICAL SENSOR ARRAY

\begin{tabular}{lccc}
\multicolumn{4}{c}{ DETAILS OF OPTICAL SENSOR ARRAY } \\
\hline \hline Transducer & Wavelength & Current measured & $\begin{array}{c}\text { Distance } \\
\text { to interrogator }\end{array}$ \\
\hline$\lambda_{1}(\mathrm{CT} 1)$ & $1535 \mathrm{~nm}$ & Infeed & $9.5 \mathrm{~km}$ \\
$\lambda_{2}$ (CT2) & $1545 \mathrm{~nm}$ & Load 2 & $14.0 \mathrm{~km}$ \\
$\lambda_{3}$ (CT3) & $1560 \mathrm{~nm}$ & Load 1 & $18.5 \mathrm{~km}$ \\
\hline \hline
\end{tabular}

\section{Reconfigurable fault thrower}

The fault throwing device used in this study is a manuallyconfigurable switch with the ability to initiate a variety of faults including three-phase, phase-to-phase, phase-to-earth, etc. In this study, the fault thrower was configured to place a phase-to-earth fault on the relevant phase for a duration of 250 ms.

There is negligible impedance associated with the fault thrower; therefore, the fault level is set by the impedance to the fault from the source. For safety reasons the fault level is limited to $70 \mathrm{~A}$ by the inclusion of the series reactance $X_{L}$ (refer to Fig. 7). Triggering and the duration of the fault is configured using dedicated software, while the fault type is determined manually at the device.

\section{Test results}

In order to demonstrate correct functionality of the proposed scheme, two sets of internal faults were applied (with differing distances between sensors), and one set of external faults was applied. The details of all applied faults are summarized in Table 2. For each internal fault scenario, the raw optical data and GRB100 disturbance records were compared directly (e.g. Fig 5) to confirm correct data transfer. However, in this section only the relay's disturbance records are shown (Figs. 9-11). Additionally, the differential current $I_{\text {diff }}$, calculated from disturbance records, is shown. During the application of external faults, only the raw optical data is recorded due to the GRB100 disturbance recorder not triggering for external faults (Fig. 12).
Due to the preliminary nature of these tests, the transducers utilized in this study exhibit a relatively high level of noise due to the non-optimum matching between the operational ranges of the ferrite-core $\mathrm{CT}$ and the piezoelectric voltage sensor. This leads to measurement resolutions during this study of $1 \mathrm{~A} \mathrm{rms}$ for the Internal 1 scenario, rising to $3 \mathrm{~A} \mathrm{rms}$ for the remaining tests after the addition of fiber reels between sensor locations. The signal-to-noise ratio can be substantially improved in future iterations of the sensors through better matching of the $\mathrm{CT}$ and piezoelectric transducer ranges.

TABLE 2

TEST SCENARIOS

\begin{tabular}{lccc}
\hline \hline Scenario & $\begin{array}{c}\text { Approx. } \\
\text { fault level } \\
\text { (A rms) }\end{array}$ & $\begin{array}{c}\text { Distance between } \\
\text { sensors } \\
(\mathrm{km})\end{array}$ & $\begin{array}{c}\text { Fault duration } \\
(\mathrm{ms})\end{array}$ \\
\hline Internal 1 & 65 & 0 & 250 \\
Internal 2 & 65 & 4.5 & 250 \\
Internal 3 & 40 & 4.5 & 250 \\
External & 40 & 4.5 & 250 \\
\hline \hline
\end{tabular}
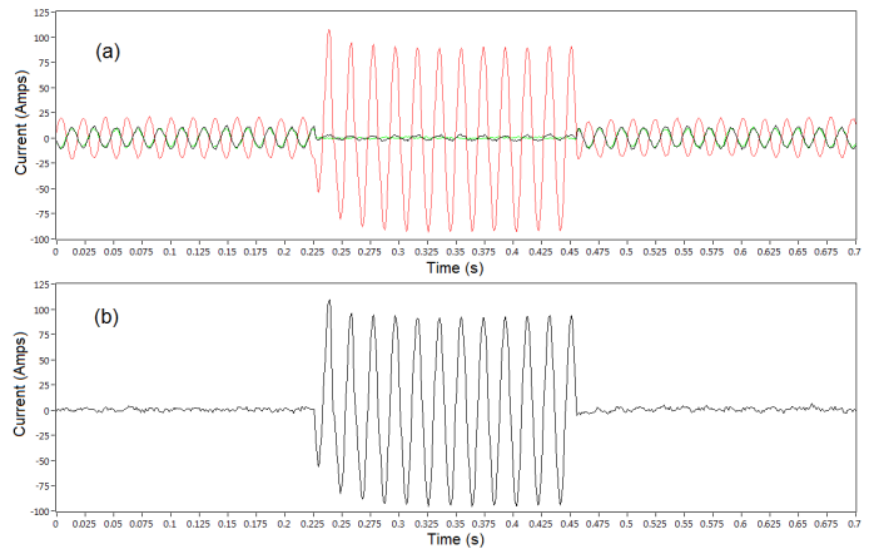

Fig. 9. (a) Relay disturbance record for internal $65 \mathrm{~A}$ rms fault under Internal 1 scenario conditions. (b) Corresponding differential current.
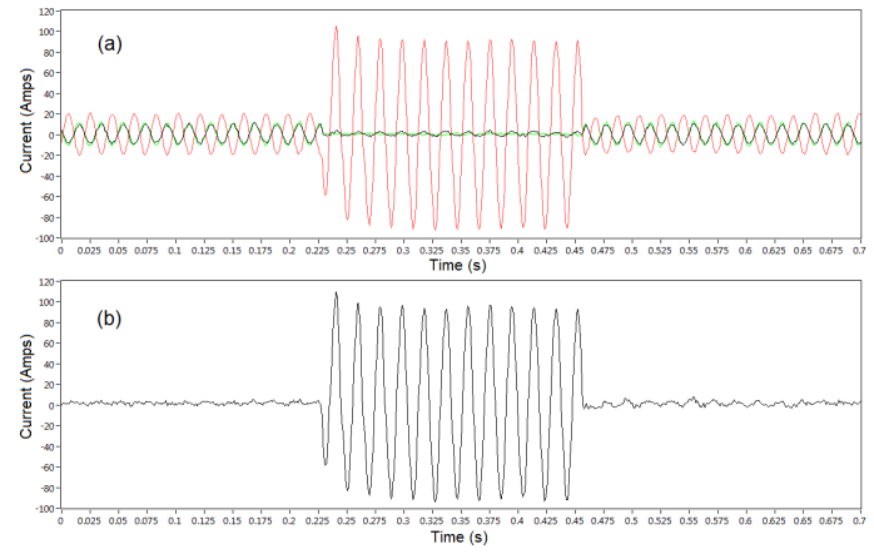

Fig. 10. (a) Relay disturbance record for internal $65 \mathrm{~A}$ rms fault under Internal 2 scenario conditions. (b) Corresponding differential current.

Regardless of present noise levels, the scheme operated correctly and in a repeatable manner for all internal and external fault scenarios applied during this study. The relay 
was able to identify the internal or external nature of the fault on $100 \%$ of occasions based purely upon measurement data from the distributed optical sensors.
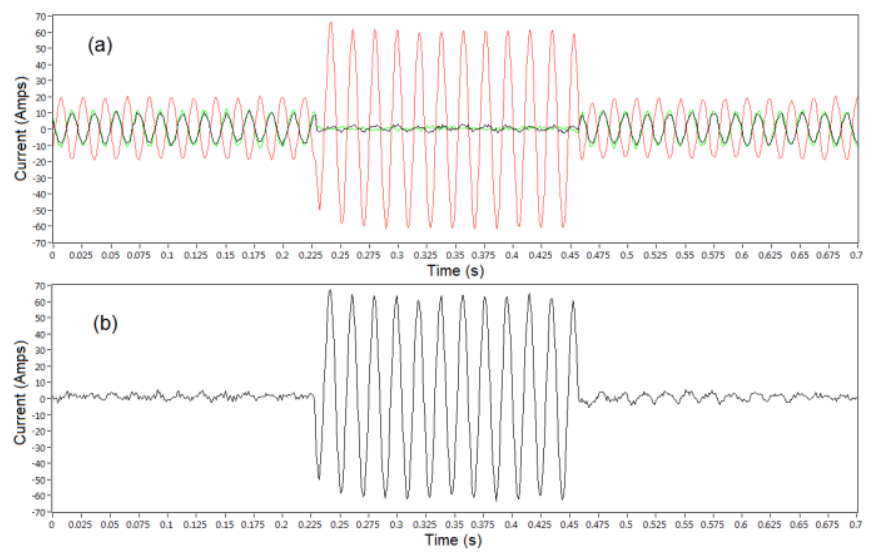

Fig. 11. (a) Relay disturbance record for internal $40 \mathrm{~A}$ rms fault under Internal 3 scenario conditions. (b) Corresponding differential current.

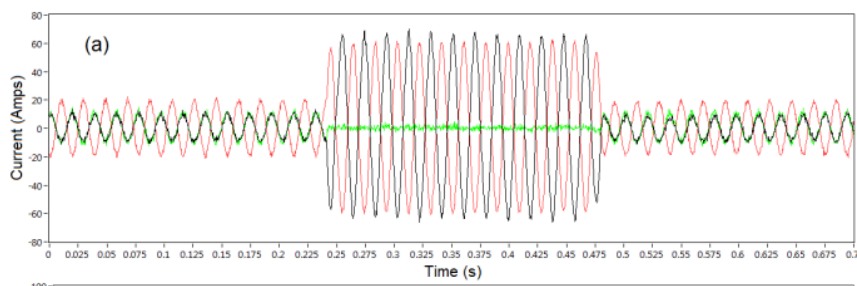

(b)

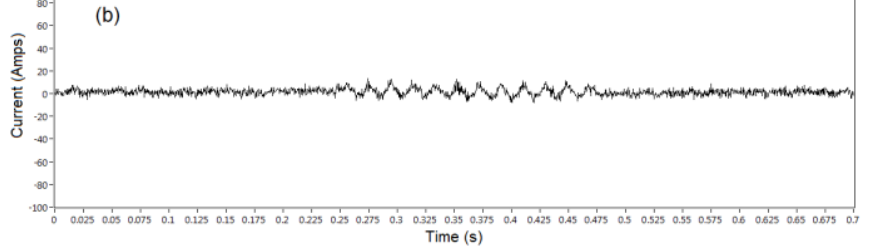

Fig. 12. (a) Optical sensor raw data recorded during $40 \mathrm{~A} \mathrm{rms} \mathrm{external} \mathrm{fault}$ scenario. (b) Corresponding calculated differential current.

\section{Discussion of Future APPLICATIONS}

A number of specific benefits to power system instrumentation may be indicated based upon the technology outlined in this paper:

- minimization of communication infrastructure;

- minimization of local measurement units and associated power sources;

- improved speed of operation owing to minimization of ADC/DAC and digital communication bottlenecks;

- flexibility in measurement location owing to the use of passive transducers addressed purely by telecomsgrade optical fiber;

- and the possibility for high visibility of multiple voltages and currents by a single central system, along with the associated potential for entirely new protection algorithms in future.

Building upon these capabilities and the demonstration presented in this article, a number of applications for this technology may be proposed.

\section{A. Protection of hybrid overhead/underground lines}

In certain network locations, hybrid lines exist, comprising a mixture of overhead line and underground cable. From a protection standpoint, it is desirable to attempt re-closure of the breaker after the fault has cleared if the fault lies on the overhead line, but not if the fault lies on the underground section. In present protection schemes, it is not possible to discriminate which section of a hybrid line is subject to the fault, and to install additional measurement and protection devices at the cable terminals is costly and can be impractical.

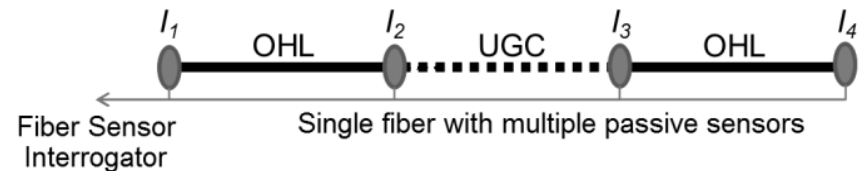

Fig. 13. Optical multi-differential (single relay) protection scheme for hybrid circuits comprising overhead line (OHL) and underground cable (UGC).

On this basis, an optical fiber multi-differential relay could be developed that would provide passive current measurement at multiple locations along the hybrid line, including at cable terminals (Fig. 13). In this way, the relay would be able to determine, with a high level of discrimination, which section of the line is faulted and could initiate (or inhibit) re-closure accordingly.

\section{B. Faster and simpler multi-terminal protection}

In Section IV of this article, the distributed optical scheme was applied to protection of a three-ended circuit with up to $4.5 \mathrm{~km}$ between sensors and a total fiber length of $18.5 \mathrm{~km}$. In this case, a single optical system replaced three separate bay units, reducing the communication and LV supply requirements. In many protection applications it will be desirable and possible to build on this initial demonstration, to increase the breadth of coverage in terms of sensor numbers and coverage area.

In contrast to present architectures, the methodology we present here has inherently less delay: Firstly, no processing or encoding is performed within the sensor network (which, importantly, may include a far greater number of sensors than conventional architectures). Since all signals remain in analog form until the interrogation unit, there is no communication bottleneck, no ADC, no processing, and physical delays set by the propagation speed of light in telecoms-grade fiber. These benefits would be particularly relevant to multi-terminal applications (Fig. 14). Such schemes presently rely on transmission of measurement data and tripping commands between many relays. Compared to the centralized optical approach, this architecture is expensive and convoluted, requiring many relays, dedicated communication channels, and sampling synchronization control. There is clear justification in this instance for development of a centralized protection scheme that can operate over wide areas with no dedicated communications infrastructure. 


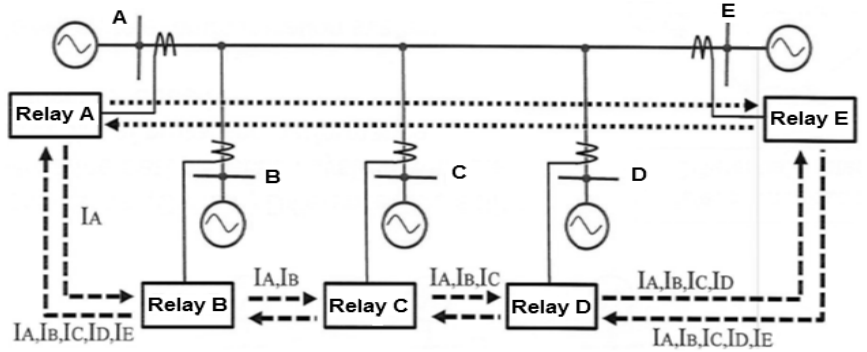

Fig. 14. A typical multi-terminal protection scheme, illustrating the processing-intensive circuital communications architecture of the scheme.

\section{UHV transformer protection}

Protection for UHV transformers (involving primary, secondary and tertiary windings) is extensive and complex due to the requirement for multiple measurement points [25]. As an example, UHV transformers may incorporate $40-50$ transducers (CTs and VTs) which must deliver measurements to the protection relay(s). The use of smaller, dielectric transducers for this purpose is desirable. In particular, the optical scheme's flexibility to multiplex all transducers on a single or multiple fibers would be highly convenient.

Additionally, FBG sensors are capable of measuring other parameters such as temperature, vibration, and pressure [4], and these measurements are therefore compatible with any chosen FBG interrogation and multiplexing scheme. A single "protection and monitoring" scheme could therefore be developed on this basis, delivering full electrical and mechanical coverage of these large, valuable items of plant.

\section{Wide-area backup protection}

Due to their capability for long-distance $(>100 \mathrm{~km})$ interrogation, FBG sensor schemes will be eminently suitable for the wide-area backup protection of multiple circuits on a large network (Fig. 15). Monitoring and protection based on voltage and current measurements taken at multiple locations will be possible from a single central location.

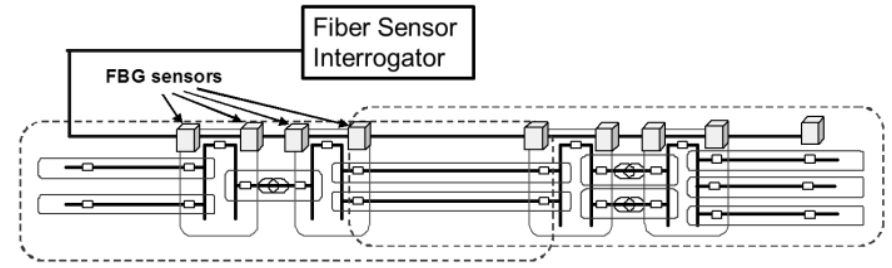

Fig. 15. Wide-area backup protection using distributed fiber sensors

\section{E. Fiber-enabled synchrophasors (without GPS)}

Presently, phasor measurement units (PMUs) require use of the GPS network to accurately time-stamp voltage or current measurements for direct comparison of phasors at diverse locations on the network [26]. Access to the GPS clock is required primarily because otherwise it is not possible to synchronize measurements that are taken by a variety of different schemes at different locations. Alternatively, the precision time protocol, which has been developed as an alternative to the GPS mechanism [27], may be applied to synchronize clocks on a network, but this requires infrastructure for continuous communication between all measurement devices.
Use of the long-distance passive fiber-optic voltage and/or current sensor network could facilitate synchrophasor measurement without the use of GPS, since in the optical scheme the relative delay between measurement points is measurable. A constant delay of approximately $4.9 \mu \mathrm{s} / \mathrm{km}(9.8$ $\mu \mathrm{s} / \mathrm{km}$ round trip) will exist between sensor reflections arriving at the interrogation unit, which can be corrected for at the central interrogator/relay. Since FBG interrogators can make measurements across a span of $100 \mathrm{~km}$ or more, there is potential for centralized wide-area synchrophasor coverage without a requirement for absolute time-stamping or access to the GPS network.

\section{F. Centralized management of smart grids}

At lower voltages, the possibility of having many current and voltage measurements available at a single location presents opportunities for the development of new protection, control and monitoring systems that will be required for future "smart" grids or active networks. It is anticipated that these networks will connect to many distributed energy sources and storage devices; thus it will be critical that protection and control systems have visibility of voltages and current throughout the networks. The approach proposed here will be ideally suited to the provision of this data, since it is able to take voltage and current measurements from several locations simultaneously. As mentioned above, use of an all-fiber approach will eliminate the need for time stamping or dedicated communications networks, which would complicate and congest future networks as the density of required measurements increases.

\section{CONCLUSIONS}

In this paper we have proposed that established optical fiber measurement technology has excellent potential to improve present power system protection systems and to enable novel protection schemes that will meet the demands of future networks. The key benefits of this technology compared with conventional electrical and existing optical techniques include passive sensing at a distance, single-fiber multiplexing, and reflection-mode operation. These benefits will facilitate reductions in the hardware and infrastructure (LV supply, telecommunications) required for wide-area protection schemes, ultimately leading to lower costs and faster operation.

The technology for voltage and current measurement using FBG-based sensors has been described, leading to a standard system design for the centralized monitoring of voltage, current, and additional compatible mechanical parameters such as temperature, strain or pressure. The integration of this measurement technology with a Toshiba GRB100 busbar protection relay has been described, and the successful deployment of the combined interrogator and relay as a threeended unit protection system was detailed. The system functioned correctly for all applied internal and external faults over a total distance of $18.5 \mathrm{~km}$ between measuring points. Even though the full potential (and limitations) of this technology is yet to be systematically quantified considering a number of protection performance influencing factors, the 
presented example provides a very realistic indication of the new capabilities.

A number of key immediate applications for the technology have been proposed based on its unique capabilities. These include combined lightweight electrical and mechanical monitoring of critical plant, centralized management of LV networks or smart grids, and synchrophasor schemes that do not require GPS radio clock access. As such, the technology is expected to have a large impact on the field of power system protection in the near future. Work is ongoing to develop high-voltage rated transducers for field demonstrations of the technology.

\section{REFERENCES}

[1] Phadke, A.G. and Thorp, J.S. (2009) Computer Relaying for Power Systems, Research Studies Press, London/John Wiley \& Sons, Inc., New York

[2] A. Moghadas, R. Barnes, M. Shadaram, "An innovative Fiber Bragg Grating sensor capable of fault detection in radial power systems," Systems Conference, 2010 4th Annual IEEE , pp.165-168, 5-8 April 2010

[3] P. Niewczas, J. R. McDonald, "Advanced optical sensors for power and energy systems," IEEE Instrumentation and Measurement Magazine, vol. 10 , no. 1 , pp. 18-28, February 2007

[4] A. D. Kersey et al, "Fiber Grating Sensors," J. Lightwave Tech., vol. 15, no. 8, pp. 1442-1463, August 1997.

[5] G. Fusiek, P. Niewczas, J. R. McDonald, "Extended Step-out Length Fiber Bragg Grating Interrogation System for Condition Monitoring of Electrical Submersible Pumps", Optical Engineering, Vol. 44, No. 3, pp 034404-1-10, March 2005

[6] Alstom NXT Phase COSI Products, http://www.nxtphase.com (Accessed 24/01/2012)

[7] K. Bonhert, P. Gabus, J. Kostovic, H. Brandle, "Optical fiber sensors for the electric power industry," Optics and Lasers in Engineering (Elsevier), vol. 43, pp. 511-526, 2005.

[8] Y. N. Ning, Z. P. Wang, A. W. Palmer, K. T. V. Grattan, and D. A. Jackson, "Recent progress in optical current sensing techniques," Rev. Sci. Instrum., vol. 66, no. 5, pp. 3097-3111, 1995

[9] Pan, F., Xiao, X., Xu, Y., and Ren, S., "An Optical AC Voltage Sensor Based on the Transverse Pockels Effect," Sensors (Basel) 11(7), 6593$6602(2011)$

[10] J. Horak and J. Hrabliuk, "Current transformer errors and transformer inrush as measured by magnetic, optical and other unconventional CTs," Available from www.basler.com/downloads/CTerrors.pdf

[11] G. Meltz, W. W. Morey, W. H. Glenn, "Formation of Bragg gratings in optical fibers by a transverse holographic method," Opt. Let., vol. 14, no. 15 , pp. 823-825, August 1989.

[12] A. Mendez, "Fiber Bragg grating sensors: a market overview," Proc. SPIE, vol. 6619, p. 661905, 2007.

[13] L. Dziuda, P. Niewczas, G. Fusiek, J. R. McDonald, "Hybrid FiberOptic Voltage Sensor for Remote Monitoring of Electrical submersible Pump Motors", Optical Engineering, Vol. 44, No. 6, pp 64401-1-6, June 2005

[14] L. Dziuda, G. Fusiek, P. Niewczas, G. Burt, and J.R. McDonald, "Laboratory Evaluation of the Hybrid Fiber-Optic Current Sensor", Sensors and Actuators, A: Physical, Vol. 136, No. 1, pp. 184-190, May 1,2007

[15] P. Niewczas, G. Fusiek, J. R. McDonald, "Dynamic capabilities of the hybrid fiber-optic voltage and current sensors", IEEE Sensors Conference, Daegu, Korea, Oct. 22-25, 2006

[16] G. Fusiek, P. Niewczas, J. R. McDonald, "Concept Level Evaluation of the Optical Voltage and Current Sensors and an Arrayed Waveguide Grating for Aero-Electrical Systems' Applications", the 24th IEEE IMTC 2007 Instrumentation and Measurement Technology Conference, Warsaw, Poland

[17] P. Orr, P. Niewczas, A. Dysko, C. Booth, "FBG-based fiber-optic current sensors for power systems protection: Laboratory evaluation," Universities Power Engineering Conference (UPEC), 2009 Proceedings of the 44th International , pp.1-5, 1-4 Sept. 2009
[18] P. Orr, P. Niewczas, A. Dysko, C. Booth, F. Kawano, G. Baber, "Distributed optical distance protection using FBG-based voltage and current transducers," IEEE Power and Energy Society General Meeting, pp. 1-5, 24-29 July 2011

[19] P. Orr, C.D. Booth, G. Fusiek, P. Niewczas, A. Dysko, F. Kawano, P. Beaumont, "Distributed Photonic Instrumentation for Smart Grids," Proc. of 2013 IEEE International Workshop on Applied Measurements for Power Systems (AMPS), Aachen, Germany, 2013

[20] P. Orr and P. Niewczas, "High-speed, solid-state, interferometric interrogator and multiplexer for fiber Bragg grating sensors," J. Lightwave Tech., vol. 29, no. 22, Nov 2011

[21] P. Orr, G. Fusiek, C.D. Booth, P. Niewczas, A. Dysko, F. Kawano, P. Beaumont, and T. Nishida, "Flexible Protection Architectures using Distributed Optical Sensors," Proc. of $11^{\text {th }}$ International Conference on Developments in Power System Protection (DPSP), Birmingham, April 2012

[22] GRB100 Busbar Protection,

http://toshiba.co.jp/sis/en/tands/protect/grb100.htm, Toshiba Corporation (Accessed 27/01/2012)

[23] R. Mackiewicz, "Overview of IEC 61850 and Benefits," in Transmission and Distribution Conference and Exhibition, 2005/2006 IEEE PES, 2006, pp. 376-383

[24] ATPS3 Protection Relay Test Set, http://www.relayeng.com/apts/apts.htm, Relay Engineering Services Limited (Accessed 27/01/2012)

[25] X. Tang, K. Kobayashi, Y. Sonobe, M. Okazaki, A. Morimoto, P. Beaumont, "Development of $765 \mathrm{kV}$ Transformer Protection Relay," Proc. Advanced Power System Automation and Protection, 2011.

[26] I. Hall, P. G. Beaumont, G. P. Baber, I. Shuto, M. Saga, K. Okuno, H. Uo, "New line current differential relay using GPS sycnhronization," Proc. IEEE Power Tech Conference, Bologna, p. 8, June 2003

[27] A. Carta, N. Locci, C. Muscas, "A PMU for the Measurement of Synchronized Harmonic Phasors in Three-Phase Distribution Networks," IEEE Trans. Instr. and Meas., vol. 58, no. 10, pp. 3723-3730, Oct 2009 